Tôhoku Math. Journ.

Vol. 18, No. 4, 1966

\title{
NOTE ON CONVERGENCE FACTORS
}

\author{
DENNIS C. RUSSELL ${ }^{1)}$
}

(Received August 9, 1966)

1. Introduction. In this paper I discuss a necessary condition on convergence factors for Riesz summability $(R, \lambda, \kappa)$ (for any $\kappa \geqq 0$ ), and also necessary and sufficient conditions on convergence factors for generalized Cesàro summability $(C, \lambda, \kappa)$ (where $\kappa$ is an integer); since (under the hypotheses used) $(C, \lambda, \kappa)$ and $(R, \lambda, \kappa)$ are equivalent, this also gives a representation for Riesz convergence factors in the case where $\kappa$ is an integer. Much attention has been given recently in work on Riesz means to the problem of imposing minimal restrictions on the sequence $\lambda$; the restriction considered here will be one which occurs naturally as a necessary condition in some circumstances, and which also appears to be capable of being used to generalize a number of existing results in which heavier restrictions on $\lambda$ have been imposed.

We suppose throughout that $\lambda=\left\{\lambda_{n}\right\}$ is a sequence satisfying

$$
0 \leqq \lambda_{0}<\lambda_{1}<\cdots<\lambda_{n} \rightarrow \infty
$$

and we shall also employ (when indicated) the condition

$$
\Lambda_{n-1}=O\left(\Lambda_{n}\right), \text { where } \Lambda_{n}=\lambda_{n+1} /\left(\lambda_{n+1}-\lambda_{n}\right)
$$

Given any series $^{2)} \Sigma a_{n}$, denote

$$
\begin{gathered}
A^{\kappa}(\omega)=\sum_{\lambda \nu<\omega}\left(\omega-\lambda_{\nu}\right)^{\kappa} a_{\nu}(\kappa \geqq 0), \quad R^{\kappa}(\omega)=\omega^{-\kappa} A^{\kappa}(\omega) ; \\
C_{n}^{0}=\sum_{\nu=0}^{n} a_{\nu}, \quad C_{n}^{p}=\sum_{\nu=0}^{n}\left(\lambda_{n+1}-\lambda_{\nu}\right) \cdots\left(\lambda_{n+p}-\lambda_{\nu}\right) a_{\nu} \quad(p=1,2, \cdots),
\end{gathered}
$$

1) This paper was written while the author was a Fellow at the Summer Research Institute of the Canadian Mathematical Congress, Kingston, Ontario, 1966.

2) When not otherwise specified, limits of summation are assumed to be 0 , $\infty$. Also $K$ will denote a constant, independent of the particular variables under consideration, and possibly different at each occurrence. We denote $\Delta b_{n}=b_{n}-b_{n+1}$. Finally, $A$ is included in $B(A \subseteq B)$ if every series summable- $A$ is also summable- $B$ (to the same value); $A$ and $B$ are equivalent $(A \sim B)$ when each is included in the other. 
(2) $g_{n}(x)=\left(1-\frac{x}{\lambda_{n+1}}\right) \cdots\left(1-\frac{x}{\lambda_{n+p}}\right)\left(0 \leqq x<\lambda_{n+1}\right), g_{n}(x)=0\left(x \geqq \lambda_{n+1}\right)$,

$$
t_{n}^{p}=\sum_{\nu=0}^{n} g_{n}\left(\lambda_{v}\right) a_{v}=\left(\lambda_{n+1} \cdots \lambda_{n+p}\right)^{-1} C_{n}^{p}
$$

$\Sigma a_{n}$ is summable $(R, \lambda, \kappa)$ to $s$ when $R^{\kappa}(\omega) \rightarrow s$ as $\omega \rightarrow+\infty$, and summable $(C, \lambda, p)$ to $s$ when $t_{n}^{p} \rightarrow s$ as $n \rightarrow \infty$. For properties of $(C, \lambda, \kappa)$ summability see Jurkat [5], Burkill [2], Russell [14], Borwein [1], Meir [16], Bcrwein-Russel [17].

If $A, B$ denote series transformations (or the matrices of such transformation's), we shall denote by $[A, B]$ the class of all summability-factors $x=\left\{x_{n}\right\}$ such that $\Sigma a_{n} x_{n}$ is summable- $B$ for every series $\Sigma a_{n}$ which is summable- $A$. When $B=I$ (convergence), $[A, I]$ is the class of all convergencefactors for $A$-summability. It is trivial that

$$
\text { if } C \subseteq A \text { then }[A, B] \subseteq[C, B]
$$

We shall require some properties of divided differences, of which an account can be found in Milne-Thomson [11], Chapter I. For non-negative integers $m, \nu, p$, denote

$$
\beta_{m v}^{(p)}=\beta_{m \nu}=\prod_{j=m}^{m+p+1}\left(\lambda_{\nu}-\lambda_{j}\right)
$$

where $\Pi^{\prime}$ indicates that any zero factor corresponding to $j=\nu$ is to be omitted. Given any function $f$ defined in the interval $\left[\lambda_{m}, \lambda_{m+p+1}\right]$, the $(p+1)$ th. order divided difference corresponding to the points $\lambda_{\nu}(m \leqq \nu \leqq m+p+1)$ is

$$
f\left[\lambda_{m}, \cdots, \lambda_{m+p+1}\right]=\sum_{\nu=m}^{m+p+1} \frac{f\left(\lambda_{\nu}\right)}{\beta_{m v}} ;
$$

if the derivative $f^{(p+1)}$ exists in $\left(\lambda_{m}, \lambda_{m+p+1}\right)$ and $f^{(p)}$ is continuous also at the end-points, we have the mean-value theorem

$$
f\left[\lambda_{m}, \cdots, \lambda_{m+p+1}\right]=\frac{1}{(p+1) !} f^{(p+1)}(\xi), \text { for some } \xi \text { in } \lambda_{m}<\xi<\lambda_{m+p+1}
$$

Also denote

$$
\gamma_{m \nu}^{(p)}=\gamma_{m \nu}=\beta_{m m} / \beta_{m \nu}
$$


so that

$$
\sum_{v=m}^{m+p+1} \gamma_{m v}=0
$$

this last result follows from (5) by taking $f(x) \equiv 1$.

LEMMA 1. Let $p$ be a non-negative integer; if $p \geqq 1$ assume that

$$
\Lambda_{n-1}=O\left(\Lambda_{n}\right)
$$

Then

$$
\left|\boldsymbol{\gamma}_{m \nu}^{(p)}\right| \leqq K\left(\Lambda_{\nu} / \Lambda_{m}\right)^{p} \quad \text { for } \quad m \leqq \nu \leqq m+p+1
$$

PROOF. For $p=0$ we have $\left|\gamma_{m \nu}^{(0)}\right|=1$ for $m \leqq \nu \leqq m+1$. If $p$ is a positive integer and $m \leqq \nu \leqq m+p+1$ then, by (4) and (6),

$$
\begin{aligned}
\left|\gamma_{m \nu}^{(p)}\right| & =\frac{\left(\lambda_{m+1}-\lambda_{m}\right) \cdots\left(\lambda_{\nu}-\lambda_{m}\right) \cdot\left(\lambda_{\nu+1}-\lambda_{m}\right) \cdots\left(\lambda_{m+p+1}-\lambda_{m}\right)}{\left(\lambda_{\nu}-\lambda_{m}\right) \cdots\left(\lambda_{\nu}-\lambda_{\nu-1}\right) \cdot\left(\lambda_{\nu+1}-\lambda_{\nu}\right) \cdots\left(\lambda_{m+p+1}-\lambda_{\nu}\right)} \\
& \leqq\left(\frac{\lambda_{m+1}-\lambda_{m}}{\lambda_{m+1}-\lambda_{m}} \cdot \frac{\lambda_{m+2}-\lambda_{m}}{\lambda_{m+2}-\lambda_{m+1}} \cdots \frac{\lambda_{\nu}-\lambda_{m}}{\lambda_{\nu}-\lambda_{\nu-1}}\right)\left(\frac{\lambda_{\nu+1}-\lambda_{m}}{\lambda_{\nu+1}-\lambda_{\nu}}\right)^{m+p-\nu+1} ;
\end{aligned}
$$

here we have merely used the fact that $\left\{\lambda_{n}\right\}$ increases, together, with the property that if $a<b<x$ then $(x-a) /(x-b)$ decreases as $x$ increases. Now if (1) holds then

$$
\text { (9) } \frac{\lambda_{m+q}-\lambda_{m}}{\lambda_{m+q}} \leqq \frac{\lambda_{m+q}-\lambda_{m+q-1}}{\lambda_{m+q}}+\cdots+\frac{\lambda_{m+1}-\lambda_{m}}{\lambda_{m+1}}=\frac{1}{\Lambda_{m+q-1}}+\cdots+\frac{1}{\Lambda_{m}} \leqq \frac{K_{q}}{\Lambda_{m}}
$$

and applying this to our last inequality for $\gamma_{m v}$ we obtain, since $\nu \leqq m+p+1$,

$$
\begin{aligned}
\left|\gamma_{m v}\right| & \leqq K\left(\frac{\lambda_{m+2} \Lambda_{m}^{-1}}{\lambda_{m+2}-\lambda_{m+1}} \cdots \frac{\lambda_{\nu} \Lambda_{m}^{-1}}{\lambda_{\nu}-\lambda_{\nu-1}}\right)\left(\frac{\lambda_{v+1} \Lambda_{m}^{-1}}{\lambda_{\nu+1}-\lambda_{\nu}}\right)^{m+p-v+1} \\
& =K \Lambda_{m}^{-p} \Lambda_{m+1} \cdots \Lambda_{\nu-1} \Lambda_{\nu}^{m+p-v+1} \\
& \leqq K \Lambda_{m}^{-p} \Lambda_{v}^{p}, \quad \text { by }(1)
\end{aligned}
$$

where $K$ is independent of $\nu$ and $m$; and this is the required result (8). 
REMARK. We note, for future reference, the following consequences of this lemma: If $p$ is a positive integer and $\Lambda_{n-1}=O\left(\Lambda_{n}\right)$ then, for $m \leqq \nu \leqq m+p+1$ :

$$
\begin{gathered}
\frac{\left(\lambda_{m+p+1}-\lambda_{m}\right) \lambda_{m+1} \cdots \lambda_{m+p}}{\left|\beta_{m \nu}^{(p)}\right|}=\frac{\lambda_{m+1} \cdots \lambda_{m+p}}{\left(\lambda_{m+1}-\lambda_{m}\right) \cdots\left(\lambda_{m+p}-\lambda_{m}\right)}\left|\gamma_{m \nu}^{(p)}\right| \\
\leqq \Lambda_{m}^{p} \cdot K\left(\Lambda_{\nu} / \Lambda_{m}\right)^{p}=K \Lambda_{\nu}^{p} \\
\frac{\lambda_{m+1} \cdots \lambda_{m+p+1}}{\left|\beta_{m \nu}^{(p)}\right|} \leqq K \Lambda_{\nu}^{p} \cdot \frac{\lambda_{m+p+1}}{\lambda_{m+p+1}-\lambda_{m}} \leqq K \Lambda_{\nu}^{p} \Lambda_{m} \leqq K \Lambda_{\nu}^{p+1}
\end{gathered}
$$

if, in addition, we have $\lambda_{\nu} \leqq t \leqq \lambda_{m+p+1}$ and $\kappa \geqq p$, then, by (9),

$$
\left(t-\lambda_{v}\right) / t \leqq\left(\lambda_{m+p+1}-\lambda_{v}\right) / \lambda_{m+p+1} \leqq K \Lambda_{v}^{-1}
$$

and hence

$$
t^{-\kappa}\left(t-\lambda_{\nu}\right)^{\kappa} \Lambda_{m}^{\kappa}\left|\gamma_{m \nu}^{(p)}\right| \leqq K\left(\Lambda_{m} / \Lambda_{\nu}\right)^{\kappa-p} \leqq K
$$

2. Convergence-factors for $(\boldsymbol{R}, \lambda, \kappa)$ summability. When the matrix $A=\left(a_{n v}\right)$ of a series-to-sequence transformation is normal (i.e. $a_{n v}=0$ for $\nu>n$, $\left.a_{n n} \neq 0\right)$, the diagonal elements of $A$ provide a limitation on the order of magnitude of the convergence-factors for $A$-summability. The following lemma is a consequence of Jurkat and Peyerimhoff [6], Satz 5.

\section{LEMMA 2a. If $A$ is normal and $x \in[A, I]$ then $x_{n}=O\left(a_{n n}\right)$.}

Normal matrices have a number of attributes which simplify consideration of their summability properties, notably the possession of a unique right inverse (which is also a left inverse). Since the $(R, \lambda, \kappa)$ method is not normal, attempts have been made to define normal methods equivalent to it (for a discussion see [14]), which sometimes necessitate restrictions on $\lambda$. Thus, for example, I have shown in [14], Theorems 4 and 5, that

$$
(C, \lambda, p) \subseteq(R, \lambda, p)(p=0,1,2, \cdots)
$$

$$
\text { if, when } p>2 \text {, (1) holds, then }(R, \lambda, p) \subseteq(C, \lambda, p)(p=0,1,2, \cdots) \text {. }
$$

(Note: Meir [16] has recently shown that $(13 \mathrm{~b})$ holds without restriction on $\lambda$ ). Again, by restricting $\omega$ in the definition of $(R, \lambda, \kappa)$ to the sequence $\left\{\lambda_{n}\right\}$, we obtain 'discrete' Riesz summability $\left(R^{*}, \lambda, \kappa\right)$ (which is normal), and Jurkat [4] has shown that 


$$
(R, \lambda, \kappa) \sim\left(R^{*}, \lambda, \kappa\right)(0 \leqq \kappa \leqq 1),
$$

without restriction on $\lambda$. Since, if $A=\left(R^{*}, \lambda, \kappa\right)$, we have $a_{n n}=\Lambda_{n}^{-\kappa}$, we obtain from (14) and Lemma 2a the result ([3], Satz 4) that

$$
\text { if } 0 \leqq \kappa \leqq 1 \text { and } x \in[(R, \lambda, \kappa), I] \text { then } x_{n}=O\left(\Lambda_{n}^{-\kappa}\right) \text {. }
$$

Maddox [8] gives an example to show that

$$
\exists \lambda, x \text { such that } x \in[(R, \lambda, 2), I] \text { but } x_{n} \neq O\left(\Lambda_{n}^{-2}\right) \text {, }
$$

so that we cannot hope to extend (15) to all $\kappa>1$ without some restriction on $\lambda$. However, Jurkat [3], Sätze 4,5, has shown that (15) remains true for $\kappa>1$ when the following conditions are imposed:

$$
\text { (a) } 0<m \leqq \frac{\Delta \lambda_{n}}{\Delta \lambda_{n-1}}, \quad \text { (b) } \frac{\Delta \lambda_{n}}{\Delta \lambda_{n-1}} \leqq M<\infty \text {. }
$$

Now if we take $A=(C, \lambda, p)$, we get

$$
a_{n n}=\left(\frac{\lambda_{n+1}-\lambda_{n}}{\lambda_{n+1}}\right) \cdots\left(\frac{\lambda_{n+p}-\lambda_{n}}{\lambda_{n+p}}\right)
$$

and if (1) holds then, by (9), $a_{n n}=O\left(\Lambda_{n}^{-p}\right)$; thus, from (13) and Lemma 2a:

$$
\begin{array}{r}
\text { if, when } p>1,(1) \text { holds, and if } x \in[(R, \lambda, p), I], \text { then } x_{n}=O\left(\Lambda_{n}^{-p}\right) \\
(n=0,1,2, \cdots) .
\end{array}
$$

The question therefore arises as to whether this result remains true with a general $\kappa$ in place of the integer $p$, and without imposing any additional restriction on $\lambda$ besides (1). It will be shown in Corollary 2 that this is in fact the case, thus showing that hypothesis (16a) can be completely removed in Jurkat's theorem ([3], Satz 4), and that (16b) can be replaced by the weaker hypothesis (1).

A further question concerns the conditions under which the sequence $\left\{\Lambda_{n}^{-\kappa}\right\}$ itself can be a convergence-factor for $(R, \lambda, \kappa)$-summability, and in considering this (at least for those values of $\kappa$ for which a normal method is known which is included in $(R, \lambda, \kappa)$ without restriction on $\lambda)$ we find that the condition (1) now appears as a necessary condition. It is convenient first to supplement Lemma $2 \mathrm{a}$ with 
LEMMA 2b. If $A$ is normal and $x \in[A, I]$ then

$$
x_{n} a_{n, n-1}=O\left(a_{n n} a_{n-1, n-1}\right) \text {. }
$$

PROOF. Denoting by $A^{-1}=\left(a_{v i}^{-1}\right)$ the two-sided inverse of $A,\left\{\sigma_{n}\right\}$ the partial sums of $\Sigma a_{v} x_{v}$, and $\left\{t_{n}\right\}$ the $A$-transform of $\Sigma a_{v}$, it is easy to deduce that

$$
\sigma_{n}=\sum_{i=0}^{n} b_{n i} t_{i}, \quad \text { where } \quad b_{n i}=\sum_{\nu=i}^{n} x_{\nu} a_{\nu i}^{-1}
$$

Thus $\left\{\sigma_{n}\right\}$ converges whenever $\left\{t_{n}\right\}$ converges (i.e. $x \in[A, I]$ ) if and only if $\left(b_{n i}\right)$ is conservative, and in particular it is necessary that

$$
\sum_{i=0}^{n}\left|b_{n i}\right| \leqq M \text { independently of } n \text {. }
$$

Hence $\left|b_{n i}\right| \leqq M$ for every $n$ and $i$; the choice $i=n$ leads at once to Lemma 2a (since $\left.a_{n n}^{-1}=1 / a_{n n}\right)$ and the choice $i=n-1$ to Lemma $2 \mathrm{~b}$, since $a_{n, n-1}^{-1}$ $=-a_{n, n-1} /\left(a_{n n} a_{n-1, n-1}\right)$.

THEOREM 1. If $0<\kappa \leqq 1$, or if $\kappa$ is a positive integer, and if

$$
\left\{\Lambda_{n}^{-\kappa}\right\} \in[(R, \lambda, \kappa), I] \text {, then } \Lambda_{n-1}=O\left(\Lambda_{n}\right) \text {. }
$$

ProOF. Let $x_{n}=\Lambda_{n}^{-\kappa}$. Then taking $A=\left(R^{*}, \lambda, \kappa\right)(\kappa>0)$ we find that

$$
\frac{x_{n} a_{n, n-1}}{a_{n n} a_{n-1, n-1}}>\left(\frac{\Lambda_{n-1}}{\Lambda_{n}}\right)^{\kappa}
$$

while taking $A=(C, \lambda, \kappa)$ ( $\kappa$ a positive integer) we obtain

$$
\frac{x_{n} a_{n, n-1}}{a_{n n} a_{n-1, n-1}}>\frac{\Lambda_{n-1}}{\Lambda_{n}}
$$

Using these inequalities in Lemma $2 b$, together with (13a), (14) and (3), the theorem follows.

While Theorem 1 gives a simple necessary condition in order that $\left\{\Lambda_{n}^{-\kappa}\right\}$ should be an $(R, \lambda, \kappa)$ convergence-factor, it is hardly to be expected that this 
condition will be sufficient, and in fact Jurkat [3], Satz 3, gives three fairly complicated sufficient conditions for such a result to hold. Taking the most tractable case for purposes of comparison, namely $\kappa=1$, we find that his first condition (which is that $\Lambda_{n} \nearrow$ ) then implies the second, while the third automatically holds, so that we have the following:

In order that $\left\{\Lambda_{n}^{-1}\right\}$ should be an $(R, \lambda, 1)$ convergence-factor, it is necessary that $\Lambda_{n-1}=O\left(\Lambda_{n}\right)$ and sufficient that $\Lambda_{n-1} \leqq \Lambda_{n}$.

However, the precise necessary and sufficient conditions in order that a sequence $\left\{x_{n}\right\}$ should be an $(R, \lambda, 1)$ convergence-factor are already given in Jurkat [3], Satz 1 (the third condition given in this theorem is superfluous, since it can be deduced from the other two, as pointed out by Maddox [10]), and if we put $x_{n}=\Lambda_{n}^{-1}$ in this theorem, we obtain:

In order that $\left\{\Lambda_{n}^{-1}\right\}$ should be an $(R, \lambda, 1)$ convergence-factor, it is necessary and sufficient that

$$
\sum_{\nu=0}^{\infty} \lambda_{\nu+1}\left|\Delta\left(\frac{\Delta \Lambda_{\nu}^{-1}}{\Delta \lambda_{\nu}}\right)\right|<\infty .
$$

We turn now to the main theorem of this section, the motivation for which has been discussed earlier.

THEOREM 2. Let $\kappa>0$. If $\kappa>1$ assume

$$
\Lambda_{n-1}=O\left(\Lambda_{n}\right) \text {. }
$$

Then for each unbounded sequence $\left\{\theta_{n}\right\}$ of real or complex numbers, there is a series $\Sigma a_{v}$, with partial sums $s_{n}$, which is summable $(R, \lambda, \kappa)$ to zero, but such that

$$
s_{n} \neq o\left(\Lambda_{n}^{\kappa} / \theta_{n}\right), \quad a_{n} \neq o\left(\Lambda_{n}^{\kappa} / \theta_{n}\right)
$$

PROOF. The proof is essentially similar to that of Jurkat [3], but here we use Lemma 1 and also alter the definition of $a_{n}$, in order to obtain sharper estimates. The choice of the series $\Sigma a_{v}$ depends on an increasing sequence of non-negative integers $\left\{n_{r}\right\}$ chosen inductively as follows: suppose that $n_{0}, n_{1}, \cdots, n_{r-1}$ have been chosen and that $a_{v}$ has been defined for $0 \leqq \nu \leqq n_{r-1}+p+1$ (where $p$ is the integer such that $p<\kappa \leqq p+1$ ) in such a way that 


$$
\sum_{\nu=0}^{n_{r-1}+p+1} a_{\nu}=0
$$

now choose $n_{r}$ so that

$$
\begin{gathered}
n_{r}>n_{r-1}+p+1 \\
\left|\theta_{n_{r}}\right| \geqq r \\
\left|\omega^{-\kappa} \sum_{\nu=0}^{n_{r-1}+p+1}\left(\omega-\lambda_{\nu}\right)^{\kappa} a_{\nu}\right| \leqq \frac{1}{r} \text { for } \omega>\lambda_{n_{r}} .
\end{gathered}
$$

Such a choice of $n_{r}$ is possible since $\left\{\theta_{n}\right\}$ is unbounded, by hypothesis, and since the left hand side of (21) tends to zero as $\omega \rightarrow \infty$, by virtue of (18). Now define

$$
\begin{gathered}
a_{\nu}=0 \text { for } n_{r-1}+p+1<\nu<n_{r} \\
a_{\nu}=\theta_{m}^{-1} \Lambda_{m}^{\kappa} \gamma_{m \nu} \text { for } m \equiv n_{r} \leqq \nu \leqq n_{r}+p+1,
\end{gathered}
$$

where $\gamma_{m v}$ is given by (6), and it then follows from (7) that (18) holds with $r+1$ in place of $r$. By making the initial choice

$$
n_{0}=0, \quad a_{\nu}=0 \text { for } n_{0} \leqq \nu \leqq n_{0}+p+1,
$$

(18)-(23) are then valid for $r=1,2, \cdots$.

It is clear that (17) holds with this choice of $\Sigma a_{\nu}$, for when $\nu=n_{r}$ we see from (22), (23) and (6) that

$$
s_{n_{r}}=a_{n_{r}}=\Lambda_{n_{r}}^{\kappa} / \theta_{n_{r}}(r=1,2,3, \cdots) .
$$

We now show that the series $\Sigma a_{\nu}$ is summable $(R, \lambda, \kappa)$ to 0 . Given $\omega>0$, let $n$ be the integer-valued function of $\omega$ satisfying $\lambda_{n}<\omega \leqq \lambda_{n+1}$; then there is an $r$ such that $n_{r} \leqq n<n_{r+1}$, and hence

$$
\begin{aligned}
\omega^{-\kappa} A^{\kappa}(\omega) & =\omega^{-\kappa} \sum_{\nu=0}^{n}\left(\omega-\lambda_{\nu}\right)^{\kappa} a_{\nu} \\
& =\omega^{-\kappa} \sum_{\nu=0}^{n_{r-1}+p+1}\left(\omega-\lambda_{\nu}\right)^{\kappa} a_{\nu}+\omega^{-\kappa} \sum_{\nu=n_{r}}^{n}\left(\omega-\lambda_{\nu}\right)^{\kappa} a_{\nu}, \text { by }(22)
\end{aligned}
$$




$$
=o(1)+S, \text { by }(21) \text {, }
$$

where

$$
S=\theta_{m}^{-1} \Lambda_{m}^{\kappa} \omega^{-\kappa} \sum_{\nu=m}^{\min (n, m+p+1)}\left(\omega-\lambda_{\nu}\right)^{\kappa} \gamma_{m \nu}\left(m=n_{r}\right)
$$

Suppose first that $m \equiv n_{r} \leqq n \leqq n_{r}+p$, so that $\omega \leqq \lambda_{m+p+1}$; if (when $p \geqq 1$ ) we assume (1), then (12) and (20) show at once that

$$
S=O\left(\theta_{n_{r}}^{-1}\right)=o(1) \quad \text { as } \quad \omega \rightarrow \infty\left(n_{r} \leqq n \leqq n_{r}+p\right)
$$

Alternatively, if $n_{r}+p+1 \leqq n<n_{r+1}$ then, by (6) and (25),

$$
\begin{aligned}
S & =\theta_{m}^{-1} \Lambda_{m}^{\kappa} \omega^{-\kappa} \beta_{m m} \cdot \sum_{\nu=m}^{m+p+1} \frac{\left(\omega-\lambda_{\nu}\right)^{\kappa}}{\beta_{m \nu}} \\
& \equiv \theta_{m}^{-1} \Lambda_{m}^{\kappa} \omega^{-\kappa} \beta_{m m} d(\omega), \text { say. }
\end{aligned}
$$

Now, by (5), $d(\omega)=e_{\omega}\left[\lambda_{m}, \cdots, \lambda_{m+p+1}\right]$, where $e_{\omega}(x)=(\omega-x)^{\kappa}$ for $\omega>x$; and it follows as in Jurkat [3], p. 270, using the mean-value theorem for divided differences, that $|d(\omega)| \leqq\left|d\left(\lambda_{m+p+1}\right)\right|$ for $\omega>\lambda_{m+p+1}$, whence

$$
|S| \leqq\left|\theta_{m}^{-1}\right| \Lambda_{m}^{\kappa} \sum_{\nu=m}^{m+p+1}\left(\frac{\lambda_{m+p+1}-\lambda_{\nu}}{\lambda_{m+p+1}}\right)^{\kappa}\left|\boldsymbol{\gamma}_{m \nu}\right|
$$

which gives, by substituting $t=\lambda_{m+p+1}$ in (12),

$$
S=O\left(\theta_{n_{r}}^{-1}\right)=o(1) \quad \text { as } \quad \omega \rightarrow \infty \quad\left(n_{r}+p<n<n_{r+1}\right) .
$$

It now follows from (24), (26), (27) that $\omega^{-\kappa} A^{\kappa}(\omega)=o(1)$ as $\omega \rightarrow \infty$; thus $\Sigma a_{v}$ is summable $(R, \lambda, \kappa)$ to zero, and the theorem is proved.

COROLLARY 2. Let $\kappa>0$; if $\kappa>1$ assume that $\Lambda_{n-1}=O\left(\Lambda_{n}\right)$. Then in order that $\Sigma a_{n} x_{n}$ should converge whenever $\Sigma a_{n}$ is summable $(R, \lambda, \kappa)$, it is necessary that $x_{n}=O\left(\Lambda_{n}^{-\kappa}\right)$. Satz 5 .

PROOF. This follows directly from the theorem, as in the proof of [3],

3. Convergence-factors for $(\boldsymbol{C}, \lambda, \kappa)$ summability. Although Lemmas 2a, $2 \mathrm{~b}$ give limitations on the order of magnitude of convergence-factors for 
$A$-summability ( $A$ normal), some more precise representations are needed in order to obtain necessary and sufficient conditions for these convergence-factors. The following result is due essentially to Peyerimhoff [12] - see also Russell [13], §2.

LemmA 3. Let $A$ be normal and $\lim _{n \rightarrow \infty} a_{n v}=1(\nu=0,1,2, \cdots)$, and let $x \in[A, I]$. Then

$$
\exists \eta,\left\{\eta_{k}\right\}, \text { with } \Sigma\left|\eta_{k}\right|<\infty \text {, such that } x_{v}=\eta+\sum_{k=v}^{\infty} \eta_{k} a_{k v}
$$

moreover,

$$
\eta_{\nu}=\sum_{k=\nu}^{\infty} x_{k} a_{k \nu}^{-1} .
$$

If, in addition, $A$ is regular (a $\gamma$-matrix) and $1 / a_{n n} \neq O(1)$, then $\eta=0$.

We obtain the last clause of the lemma as follows: if $A$ is a $\gamma$-matrix then in particular $\left|a_{k \nu}\right| \leqq K$ for every $k$ and $\nu$ and so, from (28), $x_{\nu}=\eta+o(1)$; but if $1 / a_{n n} \neq O(1)$ then, by Lemma $2 \mathrm{a}$, there is a sub-sequence of integers such that $x_{v_{s}}=o(1)$; hence $\eta=0$.

We now apply this lemma to find the form of the convergence-factors for $(C, \lambda, p)$ summability, where $p$ is an integer; there is a related result in Jurkat [5], Satz 12 - there the theorem is a consequence of a number of general results on convergence-factors, and it is interesting to see what can be proved in a direct way with fewer restrictions on $\lambda$.

THEOREM 3. Let $p$ be a non-negative integer and $g_{n}(x)$ be defined as in (2); let

$$
\Lambda_{n} \neq O(1)
$$

and when $p \geqq 2$ assume that

$$
\Lambda_{n-1}=O\left(\Lambda_{n}\right)
$$

Then $x \in[(C, \lambda, p), I]$ if and only if

$$
x_{n}=O\left\{g_{n}\left(\lambda_{n}\right)\right\}
$$




$$
\exists\left\{\boldsymbol{\eta}_{k}\right\}, \Sigma\left|\boldsymbol{\eta}_{k}\right|<\infty, \text { such that } x_{\nu}=\sum_{k=\nu}^{\infty} \eta_{k} g_{k}\left(\lambda_{\nu}\right) \text {. }
$$

PROOF. If $A$ is the $(C, \lambda, p)$ matrix, then $a_{n v}=g_{n}\left(\lambda_{\nu}\right)$ and the necessity of (31) follows at once from Lemma $2 \mathrm{a}$, without restriction on $\lambda$; however, under the hypothesis (1) (imposed for $p \geqq 2$ ), (9) shows that (31) is equivalent to

$$
x_{n}=O\left(\Lambda_{n}^{-p}\right) .
$$

In addition, $A$ is normal and regular and $1 / a_{n n} \neq O(1)$ when (30) and (1) are imposed; the necessity of (32) then follows from Lemma 3. [It should be remarked that (30) is a relatively trivial requirement, for if $\Lambda_{n}=O(1)$ then (see [14], Corollary 3B) $(C, \lambda, p) \sim I$ and the necessary and sufficient conditions for convergence-factors in this case are well-known (see, for example, Hardy [7], Theorem 7), namely: $x \in[I, I]$ if and only if $\Sigma\left|\Delta x_{n}\right|<\infty$.]

It remains to prove the sufficiency of (31) [or (31)'] and (32), and we may suppose, without loss of generality, that

$$
t_{k}^{p} \equiv \sum_{\nu=0}^{k} g_{k}\left(\lambda_{\nu}\right) a_{\nu}=o(1) \text { as } k \rightarrow \infty \text {. }
$$

Then, by (32),

$$
\begin{aligned}
\sum_{\nu=0}^{n} a_{\nu} x_{\nu} & =\sum_{\nu=0}^{n} a_{\nu}\left(\sum_{k=\nu}^{n}+\sum_{k=n+1}^{\infty}\right) \eta_{k} g_{k}\left(\lambda_{\nu}\right) \\
& \equiv \sum_{k=0}^{n} \eta_{k} t_{k}^{p}+S_{n}, \quad \text { say. }
\end{aligned}
$$

Since $t_{k}^{p}=o(1)$ and $\Sigma\left|\eta_{k}\right|<\infty$, it follows that $\Sigma \eta_{k} t_{k}^{p}$ converges, so that $\Sigma a_{\nu} x_{\nu}$ converges if and only if the sequence $\left\{S_{n}\right\}$ converges. Now

$$
S_{n}=\sum_{k=n+1}^{\infty} \eta_{k} \sum_{v=0}^{n} g_{k}\left(\lambda_{\nu}\right) a_{\nu}
$$

and by partial summation it can be shown (see Russell [14], Lemma 1) that

$$
\begin{aligned}
\sum_{\nu=0}^{n} g_{k}\left(\lambda_{\nu}\right) a_{\nu}=\sum_{r=0}^{p}( & -1)^{r} g_{k}\left[\lambda_{n+1}, \cdots, \lambda_{n+r+1}\right] C_{n}^{r}+ \\
& +(-1)^{p+1} \sum_{\nu=0}^{n} g_{k}\left[\lambda_{\nu}, \cdots, \lambda_{\nu+p+1}\right]\left(\lambda_{\nu+p+1}-\lambda_{\nu}\right) C_{\nu}^{p}
\end{aligned}
$$


For $\nu \leqq n<k-p, g_{k}\left[\lambda_{\nu}, \cdots, \lambda_{\nu+p+1}\right]$ is a $(p+1)$ th. order divided difference of a polynomial of degree $p$, and hence vanishes; consequently

$$
S_{n}=S_{n}^{\prime}+S_{n}^{\prime}
$$

where

$$
\begin{gathered}
S_{n}^{\prime}=\sum_{k=n+1}^{\infty} \eta_{k} \sum_{r=0}^{p}(-1)^{r} g_{k}\left[\lambda_{n+1}, \cdots, \lambda_{n+r+1}\right] C_{n}^{r}, \\
S_{n}^{\prime \prime}=\sum_{k=n+1}^{n+p} \eta_{k}(-1)^{p+1} \sum_{\nu=k-p}^{n} g_{k}\left[\lambda_{\nu}, \cdots, \lambda_{\nu+p+1}\right]\left(\lambda_{\nu+p+1}-\lambda_{\nu}\right) C_{\nu}^{p} .
\end{gathered}
$$

Now $g_{k}\left[\lambda_{n+1}, \cdots, \lambda_{n+r+1}\right]=\sum_{i=n+1}^{n+r+1} \frac{g_{k}\left(\lambda_{i}\right)}{\beta_{n+1, i}^{\prime}}, \quad \beta_{n+1, i}^{\prime}=\prod_{j=n+1}^{n+r+1}\left(\lambda_{i}-\lambda_{j}\right), \quad$ so that

$$
\begin{aligned}
\sum_{k=n+1}^{\infty} \eta_{k} g_{k}\left[\lambda_{n+1}, \cdots, \lambda_{n+r+1}\right] & =\sum_{i=n+1}^{n+r+1} \frac{1}{\beta_{n+1, i}^{\prime}} \sum_{k=n+1}^{\infty} \eta_{k} g_{k}\left(\lambda_{i}\right) \\
& =\sum_{i=n+1}^{n+r+1} \frac{x_{i}}{\beta_{n+1, i}^{\prime}}
\end{aligned}
$$

by (32), and since $g_{k}\left(\lambda_{i}\right)=0$ for $n+1 \leqq k<i$. Expressing $C_{n}^{r}$ in terms of $t_{n}^{r}$, it now follows from (35) that

$$
S_{n}^{\prime}=\sum_{r=0}^{p}(-1)^{r} t_{n}^{r} \sum_{i=n+1}^{n+r+1} \lambda_{n+1} \cdots \lambda_{n+r} x_{i} / \beta_{n+1, i}^{\prime}
$$

Now assuming (1) (for $r \geqq 2$ ), (11) shows (with $r-1$ in place of $p$ ) that

$$
\lambda_{n+1} \cdots \lambda_{n+r} /\left|\beta_{n+1, i}^{\prime}\right| \leqq K_{r} \Lambda_{i}^{r}(n+1 \leqq i \leqq n+r+1),
$$

and also, by (31)',

$$
x_{i}=O\left(\Lambda_{i}^{-p}\right)
$$

further (Russell [14], Corollary 3A) $t_{n}^{p}=o(1)$ implies

$$
t_{n}^{r}=o\left(\Lambda_{n}^{p-r}\right)(r=0,1, \cdots, p)
$$

Substitution of these estimates in (37) now gives, by (1), 


$$
S_{n}^{\prime}=\sum_{r=0}^{p} \sum_{i=n+1}^{n+r+1} o\left(\Lambda_{n}^{p-r} \Lambda_{i}^{r} \Lambda_{i}^{-p}\right)=o(1) .
$$

Turning to $S_{n}^{\prime \prime}$, we note first that $S_{n}^{\prime \prime}$ vanishes identically for $p=0$ and $p=1$. Now $g_{k}\left(\lambda_{i}\right)=0$ for $k<i \leqq \nu+p+1$, so that

$$
g_{k}\left[\lambda_{\nu}, \cdots, \lambda_{\nu+p+1}\right]=\sum_{i=v}^{k} \frac{g_{k}\left(\lambda_{i}\right)}{\beta_{v i}}, \quad \beta_{\nu i}=\prod_{j=\nu}^{\nu+p+1}\left(\lambda_{i}-\lambda_{j}\right)
$$

Now (9) shows, making use of (1), that

$$
g_{k}\left(\lambda_{i}\right)=\frac{\left(\lambda_{k+1}-\lambda_{i}\right) \cdots\left(\lambda_{k+p}-\lambda_{i}\right)}{\lambda_{k+1} \cdots \lambda_{k+p}} \leqq K \Lambda_{i}^{-p} \quad \text { for } \quad k-p \leqq i \leqq k
$$

Also

$$
\frac{\left(\lambda_{\nu+p+1}-\lambda_{\nu}\right)\left|C_{\nu}^{p}\right|}{\left|\beta_{v i}\right|}=\frac{\left(\lambda_{\nu+p+1}-\lambda_{\nu}\right) \lambda_{\nu+1} \cdots \lambda_{\nu+p}\left|t_{\nu}^{p}\right|}{\left|\beta_{v i}\right|} \leqq K \Lambda_{i}^{p}
$$

for $\nu \leqq i \leqq \nu+p+1$, by (10) (assuming (1)) and since $\left\{t_{\nu}^{p}\right\}$ is bounded, by hypothesis. Substituting these estimates into (26), we now find that

$$
\left|S_{n}^{\prime \prime}\right| \leqq K \sum_{k=n+1}^{n+p}\left|\eta_{k}\right|=o(1)
$$

Hence, by (34), (38), (39), we have $S_{n}=o(1)$ and so, by (33), $\sum a_{\nu} x_{\nu}$ converges to $\Sigma \eta_{k} t_{k}^{p}$; and this proves the theorem.

To write Theorem 3 in an alternative form, an easy calculation shows that the two-sided inverse matrix $A^{-1}=\left(a_{k v}^{-1}\right)$ of the $\gamma$-matrix $A=(C, \lambda, p)$ is given by

$$
\begin{gathered}
a_{k v}^{-1}=(-1)^{p+1}\left(\lambda_{\nu+p+1}-\lambda_{v}\right) \lambda_{v+1} \cdots \lambda_{\nu+p} / \beta_{v k} \quad(\nu \leqq k \leqq \nu+p+1), \\
a_{k v}^{-1}=0 \text { otherwise, }
\end{gathered}
$$

where $\beta_{v k}$ is defined by (4). Thus $A^{-1}$ consists of $p+2$ diagonals containing non-zero elements, with zero elements elsewhere. Now using (29), Theorem 3 takes the form:

THEOREM 3'. Under the hypotheses of Theorem $3,\left\{x_{n}\right\}$ is a $(C, \lambda, p)$ convergence-factor if and only if 


$$
\begin{gathered}
x_{n}=O\left(\Lambda_{n}^{-p}\right) \\
\sum_{n=0}^{\infty}\left(\lambda_{n+p+1}-\lambda_{n}\right) \lambda_{n+1} \cdots \lambda_{n+p}\left|\sum_{i=0}^{p+1} \frac{x_{n+i}}{\beta_{n, n+i}}\right|<\infty .
\end{gathered}
$$

For $\lambda_{n}=n$, the inner sum in (41) reduces to $(-1)^{p+1}(p+1) ! \Delta^{p+1} x_{n}$, and we obtain as a corollary the well-known Bohr-Hardy-Fekete theorem :

COROLLARY 3'. Let $p$ be a non-negative integer; then $\Sigma a_{n} x_{n}$ converges whenever $\Sigma a_{n}$ is summable $(C, p)$ if and only if $x_{n}=O\left(n^{-p}\right)$ and $\Sigma n^{p+1}\left|\Delta^{p+1} x_{n}\right|$ $<\infty$.

This result has been generalized in several directions, notably by Bosanquet and Andersen; for further references and a short discussion of convergencefactors for Cesàro summability see Hardy [7], p. 146.

Finally, we remark that in view of $13(\mathrm{a}),(\mathrm{b})$, Theorems 3 and $3^{\prime}$ also give (for integral $p$ ) necessary and sufficient conditions in order that $\Sigma a_{n} x_{n}$ should converge whenever $\Sigma a_{n}$ is summable $(R, \lambda, p)$. By restricting $\lambda$ to satisfy (16a) together with $\Lambda_{n} \nearrow+\infty$ (which implies (16b)) Maddox [8] has been able to obtain a considerably more general result for summability-factors $[(R, \lambda, \kappa)$, $(R, \lambda, \mu)]$; there the summability-factors are expressed in the form of an integral instead of a series form such as (32). The precise relation between the two forms would be quite difficult to determine, though Maddox [9] gives an interesting construction in the case $\lambda_{n}=n$, i.e. for $[(C, \kappa),(C, \mu)]$ summabilityfactors. The problem is also mentioned by Jurkat and Peyerimhoff [6], p. 105, in comparing (for $0<\kappa \leqq 1$ ) the integral form for $(R, \lambda, \kappa)$ convergence-factors with the series form for $\left(R^{*}, \lambda, \kappa\right)$ convergence-factors. Recently I have been able to show (see [15]) that the conditions on $\lambda$ imposed by Maddox [8], Theorem $\mathrm{A}$, in his result on $[(R, \lambda, \kappa),(R, \lambda, \mu)]$ summability-factors, can be removed entirely in the case $0 \leqq \mu \leqq \kappa \leqq 1$.

\section{REFERENCES}

[1] D. BoRwEIN, On a generalized Cesàro summability method of integral order, Tôhoku Math. Journ., (2), 18(1966), 71-73.

[2] H. BuRkill, On Riesz and Riemann summability, Proc. Camb. Phil. Soc., 57(1961), $55-60$.

[3] W. B. JuRKat, Über Konvergenzfaktoren bei Rieszschen Mitteln, Math. Zeit., 54(1951), 262-271.

[4] W. B. JuRKat, Über Rieszsche Mittel mit unstetigem Parameter, Math. Zeit., 55(1951), $8-12$.

[5] W. B. JURKAT, Über Rieszsche Mittel und verwandte Klassen von Matrixtransformationen, Math. Zeit., 57(1953), 353-394. 
[6] W. B. Jurkat AND A. Peyerimhoff, Mittelwertsätze bei Matrix- und Integraltransformationen, Math. Zeit., 55(1951), 92-108.

[7] G. H. HARDy, Divergent Series, Oxford University Press (1949).

[8] I. J. MADDOX, Convergence and summability factors for Riesz means, Proc. London Math. Soc. (3), 12(1962), 345-366.

[9] I. J. MADDOX, A note on summability factor theorems, Quart. Journ. Math. (Oxford) (2), 15(1964), 208-216.

[10] I. J. MADDOX, Some inclusion theorems, Proc. Glasgow Math. Assn. 6(1964), 161-168.

[11] L. M. Milne-Thomson, The calculus of finite differences, Macmillan, London (1933, reprinted 1960).

[12] A. PeYERIMHOFF, Konvergenz- und Summierbarkeitsfaktoren, Math. Zeit., 55(1951), 23-54.

[13] D. C. Russell, Note on inclusion theorems for infinite matrices, Journ. London Math. Soc., 33(1958), 50-62.

[14] D. C. Russell, On generalized Cesàro means of integral order, Tôhoku Math. Journ. (2), 17(1965), 410-442. Corrigenda; 18(1966), 454-455.

[15] D. C. Russell, On a summability factor theorem for Riesz means, Journ. London Math. Soc. (to appear).

[16] A. MEIR, An inclusion theorem for generalized Cesàro and Riesz means, Canada. Journ. Math. (to appear).

[17] D. BORWEIN AND D. C. RUSSEL, On Riesz and generalized Cesàro summability of arbitrary positive order, Math. Zeit. (to appear).

YORK UNIVERSITY.

TORONTO 12, CANADA. 